\title{
Guía de Práctica Clínica Manejo a largo plazo de las conductas autolesivas
}

\author{
Self-harm: longer-term management (NICE clinical guideline 133)
}

Resumido y traducido con autorización del National Institute for Health and Clinical Excellence (NICE). Disponible en: http://guidance.nice.org.uk/CG133

\section{Introducción}

Las autolesiones son los daños provocados por una persona sobre su propio cuerpo (por ejemplo, cortes, pinchazos, quemaduras, etc.) y son conductas que se observan con frecuencia en la práctica psiquiátrica. Si bien un estudio realizado en 17 países informó que el $2,7 \%$ de los adultos había presentado autolesiones, su prevalencia podría estar subestimada debido a que la mayoría de los estudios están basados en autoreportes.

La asociación entre este tipo de conductas y la presencia de un trastorno psiquiátrico es contundente. En este sentido, el trastorno límite de la personalidad (TLP) es el que presenta la mayor frecuencia ${ }^{1}$ y se podría decir que constituye una de las características que lo distingue.

Un paciente que se autolesione presenta un riesgo de suicidio 50 a 100 veces mayor que el de la población general. Por lo tanto, estar atentos a la presencia de este tipo de conductas autolesivas es un aspecto fundamental en la prevención del suicidio.

Esta guía de práctica clínica resume las recomendaciones publicadas por el National Institute for Health and Clinical Excellence (NICE) referidas al manejo a largo plazo de aquellos pacientes que se provocan lesiones. Constituye un complemento de la guía de NICE publicada previamente sobre el tratamiento de las autolesiones producidas a corto plazo (dentro de las 48 horas).

\section{Consideraciones generales}

Los aspectos que deben tenerse en cuenta cuando se trabaja con personas que se provocan lesiones son:

- Generar un vínculo terapéutico de confianza. Esto estimulará al paciente a exponer su problemática y sus emociones y le permitirá al médico recabar datos relevantes de la historia clínica y llevar a cabo una derivación específica y adecuada. Además, de este modo, se favorecerá el cumplimiento de las sugerencias de un tratamiento ${ }^{2}$.

- Procurar no estigmatizar al paciente ni relativizar su motivo de consulta. Es importante manifestar preocupación al respecto. - Evitar adoptar una actitud paternalista. Siempre que sea posible, es preferible estimular la toma de decisiones por parte del paciente y ayudarlo a que se involucre en el abordaje su dolencia.

- Mantener la relación terapéutica continua en la medida de lo posible, más allá de que se haya llevado a cabo una derivación a un tratamiento específico. En caso contrario el paciente puede interpretar esto como un abandono.

- Asegurar una comunicación entre los miembros del equipo tratante con respecto a la conducta autoagresiva.

\section{La evaluación del especialista}

Ante un paciente con conductas autolesivas y haciendo foco en las particularidades de cada grupo etáreo, el psiquiatra trabaja básicamente sobre tres niveles diagnósticos:

- La presencia o no de un trastorno psiquiátrico y/u otras comorbilidades clínico-neurológicas.

- La personalidad del paciente (mecanismos de defensa, fortalezas, estrategias de afrontamiento, etc.).

- El contexto familiar, la situación laboral/ocupacional y económica, los problemas psicosociales que requieran una intervención específica (por ejemplo, tener hijos a cargo) y la presencia de estresores vitales como duelos, nacimientos, mudanzas, enfermedad de allegados o jubilación.

Los factores que debe tenerse en cuenta cuando se evalúa pacientes con autolesiones son:

- El método utilizado habitualmente y la frecuencia, tanto en el pasado como en el presente.

- Los antecedentes de intentos de suicidio. Si bien en general es difícil discriminar entre una autolesión y un intento de suicidio, autores como Gunderson ${ }^{3}$ recomiendan no ignorar ni relativizar episodios de autoagresión en los pacientes con trastorno límite de la personalidad ya que un suicidio consumado puede ocurrir cuando existe un error de cálculo relacionado con un rescate que se esperaba.

- La presencia de sintomatología depresiva o de otro trastorno psiquiátrico.

- El sostén familiar.

- El cumplimiento (si lo hubiera) del tratamiento actual.

- Los factores desencadenantes a nivel personal, familiar, laboral, etc.

- Los factores de riesgo y protectores sociales, psicológicos, farmacológicos y motivacionales que puedan incrementar o evitar un episodio autolesivo.

- El riesgo inmediato y la necesidad de internación.

No existe evidencia para recomendar el uso de escalas de evaluación de riesgo suicida o de aquellas que predicen la repetición de un episodio autolesivo.

\section{Recomendaciones para el cuidado del paciente}

Los objetivos de un plan de cuidado para un paciente con episodios de autolesiones son:

- Prevenir el aumento de la frecuencia y gravedad de las autolesiones. - Reducir o detener los daños provocados, tanto físicos como sociales (por ejemplo, internaciones repetidas que repercuten en la vida social y laboral).

- Reducir el riesgo de suicidio.

- Mejorar el funcionamiento social u ocupacional y la calidad de vida del paciente y su familia con metas realistas y optimistas a largo plazo.

Es fundamental revisar estos objetivos junto con el paciente, su familia, el equipo interdisciplinario y toda persona considerada significativa a los fines del tratamiento, identificando los roles que cada uno cumple y asignándoles responsabilidades.

\section{Manejo del riesgo y del episodio autolesivo}

Ante un paciente con el riesgo de autodaño o con episodio autolesivo es importante considerar las siguientes intervenciones para prevenir la repetición del episodio y disminuir el riesgo suicida.

- Permitir que el paciente relate lo que le sucede, expresando el terapeuta preocupación al respecto.

- Preguntar si desea ayuda y qué tipo de ayuda desea recibir, ofreciéndole realizar un seguimiento.

- Tratar de identificar los desencadenantes y discutir los aspec- 
tos relacionados con el cuidado.

- Interpretar las razones que proporcionaron el alivio y sus motivos.

- Evidenciar la imposibilidad de disponer del terapeuta en todo momento y trabajar sobre las alternativas posibles.

- No considerar al tratamiento farmacológico como una intervención específica para reducir las autolesiones.

- Identificar y tratar al trastorno psiquiátrico asociado a la conducta autoagresiva: alcoholismo, depresión, esquizofrenia, trastorno límite de la personalidad, trastorno bipolar.

Se debe recordar que la intervención terapéutica debe adaptarse a las necesidades individuales y ser consistente con las estrategias del tratamiento a largo plazo.

\section{Contexto y dificultades}

Los servicios encargados de la atención de los pacientes que se autolesionan pueden tener diferentes características. Unos proporcionan un tratamiento integral y ofrecen la capacitación profesional pero otros solo se limitan a un servicio de urgencias. Entre los motivos de estas diferencias se destaca la falta de recursos que se destinan para el tratamiento de este tipo de pacientes, dado que en general los resultados obtenidos son pobres y las recaídas, frecuentes. Esto es importante si se tiene en cuenta que ante una atención inadecuada, aumenta la probabilidad de que el paciente evite una consulta en el futuro, con el consiguiente aumento de su riesgo suicida.

Esta guía tiene como objetivo brindar el conocimiento sobre el manejo de estas situaciones y alertar sobre esta problemática. Además, procura evitar el estigma al que se ven sometidos estos pacientes, lo que dificulta su acceso a una evaluación y a tratamientos psicológicos y psiquiátricos adecuados.

Si tenemos en cuenta que los episodios de autolesiones constituyen un factor de riesgo suicida importante, es importante no relativizarlos.

Fuente de financiamiento: National Institute for Heatlth and Clinical Excellence.

Nota: las guías NICE son desarrolladas para su aplicación dentro del Servicio Nacional de Salud (sigla en ingles NHS) en el Reino Unido. El NICE no toma responsabilidad alguna por la traducción al español aquí publicada de la presente guía.

\section{Comentario}

Las recomendaciones que brinda la guía NICE que hemos reproducido son respecto a un signo-síntoma común en la práctica psiquiátrica: las autolesiones. Sin embargo, consideramos que esta guía ha dejado de lado el contexto en el que generalmente se producen este tipo de episodios: pacientes con trastorno límite de la personalidad (o todos los incluidos en el grupo B del trastorno de personalidad según el Manual Estadístico de Trastornos Mentales, en inglés DSM-IV y desde ahora TLP). Esto no quiere decir que en otros cuadros no se puedan producir conductas de autoagresión, pero predominan en los pacientes con TLP y como afirman los autores de la guía: “...la personalidad límite parece estar caracterizada por el comportamiento autoagresivo crónico, incluido el comportamiento altamente letal..."1. Por este motivo, considero importante profundizar en ello. Lamentablemente, faltan aún indicios certeros sobre qué conductas deben considerarse intentos suicidas reales y las estadísticas, tampoco son tranquilizadoras ya que estas conductas tienen un alto índice de mortalidad secundaria. Vale destacar que $10 \%$ de estos pacientes se suicidan, lo que representa un riesgo 50 veces mayor que el de la población general ${ }^{4}$.

Por esto, la evaluación del riesgo suicida en los pacientes con TLP es una de las tareas más importantes y de mayor responsabilidad dentro de la práctica psiquiátrica, lo que hace que sea fundamental tener en cuenta los probables factores de riesgo y poseer una buena técnica de entrevista con el fin de obtener la información más completa posible y optimizar la exploración del paciente.

Por otro lado, no existe ningún factor o combinación de factores de riesgo que tenga una sensibilidad y especificidad suficiente para predecir el paso al acto, por lo que debemos optimizar la única herramienta con la que contamos para prevenir el suicidio: la evaluación clínica.

Al evaluar el potencial suicida, se deben valorar las motivaciones del paciente, la accesibilidad a cometer el acto, el estado de sus relaciones interpersonales y sus antecedentes. Desde una perspectiva psicodinámica hay que tener en cuenta que ante una eventual pérdida de algún apoyo, o en respuesta a no recibir cuidados suficientes, se puede incrementar el riesgo de una autolesión.

Si bien el antecedente de episodios previos de autolesión suma gravedad al actual, no consideramos pertinente la separación entre un manejo a corto y otro a largo plazo, debido a que la urgencia queda constituida al producirse la autolesión.

\section{Conclusiones del comentador}

Las lesiones autoprovocadas pueden ser la puerta de entrada a un tratamiento psiquiátrico. Su presencia es un indicador de gravedad del paciente y no se las debe relativizar ni estigmatizar. La entrevista es la herramienta principal tanto para el diagnóstico, como para el desarrollo de un vínculo terapéutico, constituyendo un verdadero desafío el manejo de los pacientes con autolesiones. Por eso es importante tener presente que el tratamiento siempre debe ser interdisciplinario y con una buena comunicación entre los miembros del equipo tratante.

\section{Hugo Pisa [ Servicio de Psiquiatria de Hospital Italiano de Buenos Aires. hugo.pisa@ hospitalitaliano.org.ar ]}

Pisa H. Manejo a largo plazo de las conductas autolesivas. Evid Act Pract Ambul. Jul-Sep 2013;16(3):96-97. Resumido, traducido y comentado de: National Institute for Health and Clinical Excellence. Self-harm: Ionger-term management (NICE clinical guideline 133). Disponible en: http://guidance.nice.org.uk/CG133.

\section{Referencias}

1. Sansone R y col. Aproximaciones diagnósticas a la personalidad borderline y su relación con la conducta autolesiva. Int J Psychiatr Clin Pract (Ed. Esp) 2002; 1: 95-100.

2. Hillard J y col. Suicide in a psychiatric emergency room population. Am J Psychiatry 1983; 140: 459-462.

3. Gunderson J. Trastorno límite de la personalidad. Ed. ARS Médica. Barcelona. 2002.

4. Klaus L y col. Borderline personality disorder. Lancet 2004; 364: 453-61. 